\title{
Minimizing 6-fold Symmetry and Chromatic Aberration by using Ultra High Resolution Lens in Cs-Corrected STEM
}

\author{
K. Nakamura,* H. Inada, ${ }^{*}$ M. Konno,* Y. Suzuki* and K. Kimoto** \\ *Hitachi High Technologies Corp., Ibaraki, Japan \\ **National Institute for Material Science, Ibaraki, Japan
}

Ultra high resolution HAADF-imaging and chemical analysis in the field of material science and nano-technology has been demonstrated by using Cs (spherical aberration)-corrected STEM. Also detecting single atoms by secondary electron imaging with Cs-corrected Cold FEG-STEM is reported recently [1]. In this field, Cs-corrected tool allow us to obtain $0.1 \mathrm{~nm}$ in daily use.

Multi-pole type corrector is the most feasible tool to compensate the spherical aberration, however, the higher order aberration is introduced by the corrector. For example, Hexapole type corrector introduces 6-fold astigmatism (A5) in high angle component [2]. Chromatic aberration is more effective factor because chromatic aberration at $200 \mathrm{kV}$ by using $24 \mathrm{mrad}$-convergence angle is around $0.1 \mathrm{~nm}$ in case of Schottky emitter $(\mathrm{Cc}=1.7 \mathrm{~mm}$ is assumed). To get sub-angstrom resolution routinely, we tried to minimize the A5 and chromatic aberration by using cold field emission gun (CFEG) and ultra high resolution lens (the original $\mathrm{Cs}$ and $\mathrm{Cc}$ is around $0.55 \mathrm{~mm}$ and $1.1 \mathrm{~mm}$, respectively). This idea is to reduce residual A5 by decreasing Hexapole strength with small change of focal length.

Figure 1 shows calculated root mean square of residual aberrations including diffraction limit, chromatic aberration and 6-fold astigmatism with respect to UHR, HR and analysis objective lens at $200 \mathrm{kV}$. The energy spread of $0.3 \mathrm{eV}$ for CFEG is used. The cumulative aberration shows $78 \mathrm{pm}$, $68 \mathrm{pm}$ and $63 \mathrm{pm}$ for analysis, HR and UHR lens, respectively. This theoretical consideration supports the feasibility of UHR lens, and also means the other residual aberration should be eliminate completely to acquire $63 \mathrm{pm}$-resolution at $200 \mathrm{kV}$.

Figure 2 shows Ronchigrams taken with (a) HR lens and (b) UHR lens at 200kV. The 6-fold symmetry reduced at UHR lens in contrast to HR lens, and feature-free region extends from 35(HR lens) to $44 \mathrm{mrad}$ (UHR lens). This result means that UHR lens is effective to reduce A5 and chromatic aberration.

\section{References}

[1] Y. Zhu, H. Inada, K. Nakamura, and J. Wall, Nature Materials, 8808 (2009).

[2] H. Mueller, S. Uhlemann, P. Hartel, and M. Haider, Microsc. Microanal. 12, 442 (2006). 


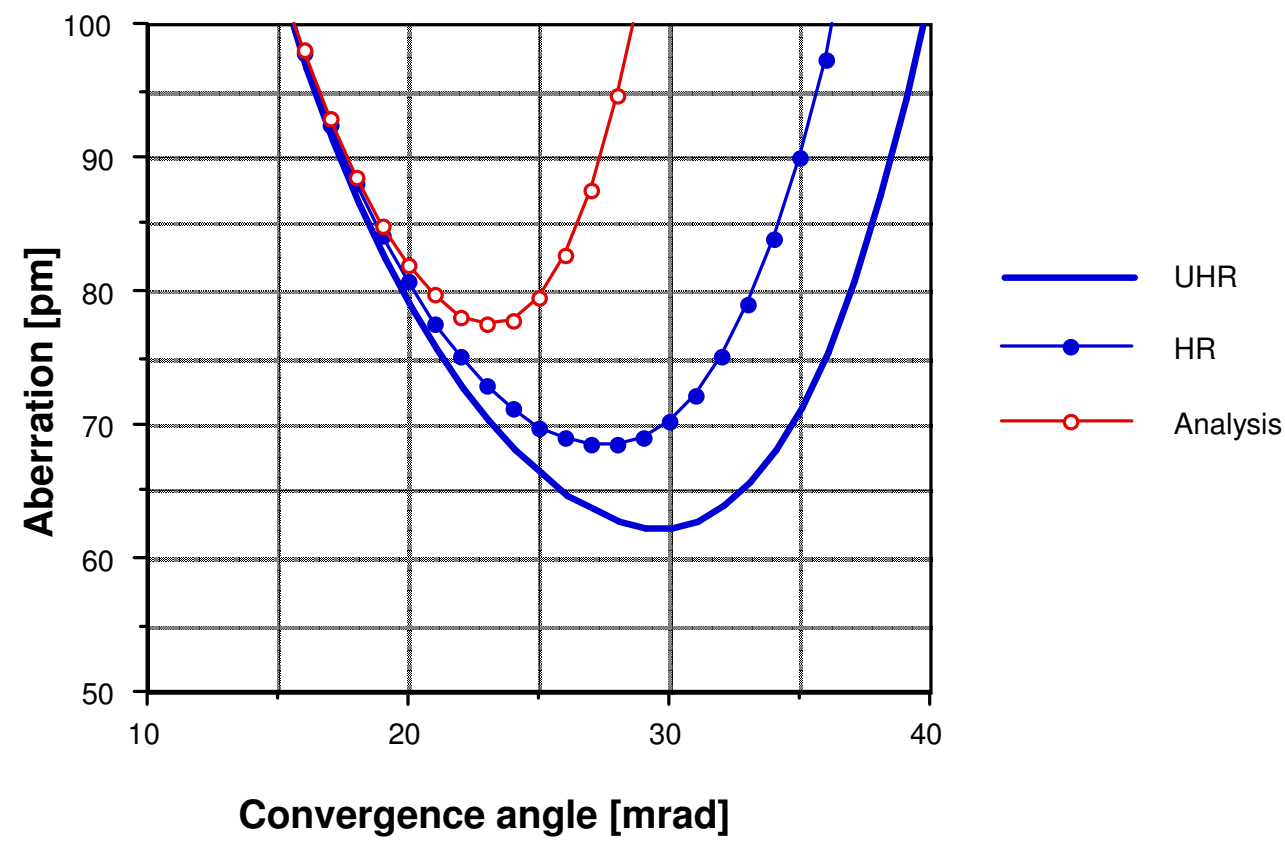

Fig.1 Calculated root mean square of residual aberrations including diffraction limit, chromatic aberration and 6-fold astigmatism with respect to UHR, HR and analysis objective lens at 200kV.
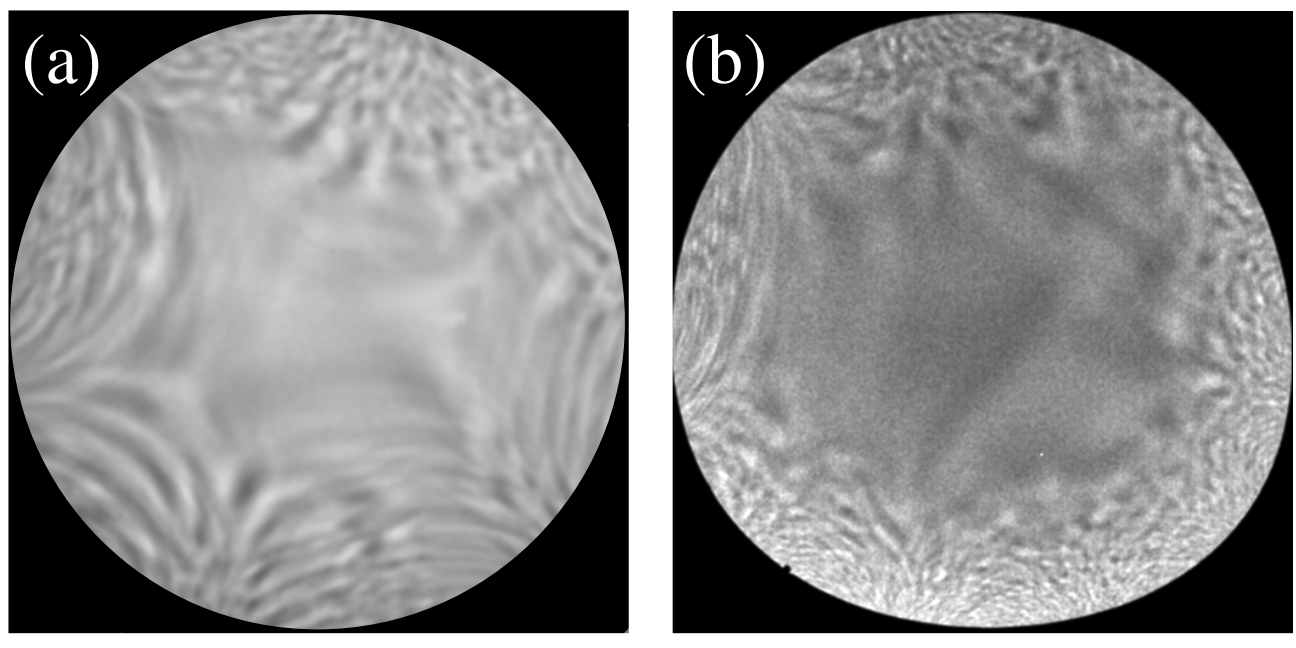

$30 \mathrm{mrad}$

Fig.2 Ronchigrams taken with (a) HR lens and (b) UHR lens at 200kV. 